\title{
Letter to the editor on the article "Treatment of grade III and IV haemorrhoidal disease with PPH or THD. A randomized trial on postoperative complications and short-term results"
}

\author{
Jin-Yao Teo • Ming-Hian Kam $•$ Kong-Weng Eu
}

Accepted: 21 May 2010/Published online: 9 June 2010

(C) Springer-Verlag 2010

\section{Dear Editor:}

We refer to the article entitled "Treatment of grade III and IV haemorrhoidal disease with PPH or THD. A randomized trial on postoperative complications and short-term results" published in December 2009. While we congratulate the authors on publishing their results of a randomized controlled trial on what is a new and exciting treatment modality for haemorrhoidal disease, we would like to share our thoughts on the subject.

The authors set out to compare Procedure for Prolapsed Haemorrhoids (PPH) with Transanal Haemorrhoidal Dearterialisation (THD) with regards to post-operative complications and short-term results. They used "resolved pre-operative symptoms with no need for further treatment" as a primary end-point. We feel that this is not objective enough. For grade III and IV haemorrhoids, one of the main symptoms would be that of prolapse. Here, the authors have not quantified the nature of prolapse nor investigated the extent of resolution of the pre-operative prolapse after surgery. Further, it appears that there is only one patient in the PPH arm with grade IV haemorrhoids, compared with four patients in the THD arm. Could the observed difference in persistent prolapse between the two arms at 6 weeks be due to this?
Overall, the small sample size suggests that the study may be inadequately powered to show a true difference between the two treatment modalities. It would have been helpful to include information on power calculations. With respect to the technical details of surgery, it was unclear how exactly THD was performed. It would also be helpful if the authors clarified whether three-, four- or six-point ligation was performed. In addition, was a plicating stitch or anopexy routinely performed in all cases? Finally, we wish to point out that this study did not look specifically into the costs and learning curve for performing both procedures. As such, the last statement that THD is "more easily learned and less costly" remains to be proven.

In our ongoing study of 140 patients who had undergone THD, we have found persistent prolapse at 6 weeks in $19.2 \%$ of patients with grade III piles. This compares with $22.2 \%$ of patients in the current study who complained of persistent prolapse at 6 weeks. The authors mentioned that, in their PPH arm, only $11.1 \%$ had persistent prolapse. There appears to be twice the risk of persistent prolapse if grade III or IV haemorrhoids were operated on using THD instead of PPH. Based on the results reported in this study, perhaps we should only recommend THD for haemorrhoidal bleeding and not prolapse at this point in time.

J.-Y. Teo • M.-H. Kam • K.-W. Eu ( $\square)$

Department of Colorectal Surgery,

Singapore General Hospital,

Outram Road,

Singapore 169608, Singapore

e-mail: the_gentleman82@hotmail.com 